\section{() OPEN ACCESS}

\title{
Gut-focused hypnotherapy for children and adolescents with irritable bowel syndrome
}

\author{
Dipesh H Vasant (D) , ${ }^{1,2}$ Syed S Hasan, ${ }^{1}$ Pamela Cruickshanks, ${ }^{1}$ \\ Peter J Whorwell ${ }^{1,2}$
}

- Additional material is published online only. To view please visit the journal online (http://dx.doi.org/10.1136/ flgastro-2020-101679).

'Gastroenterology Department, Neurogastroenterology Unit, Wythenshawe Hospital, Manchester University NHS Foundation Trust, Manchester, UK ${ }^{2}$ Division of Diabetes, Endocrinology and Gastroenterology, University of Manchester, Manchester, UK

\section{Correspondence to}

Dr Dipesh H Vasant, Neurogastroenterology Unit, Manchester University NHS Foundation Trust, Manchester, Greater Manchester, UK; dipesh. vasant@manchester.ac.uk

Received 9 September 2020 Revised 13 October 2020 Accepted 29 October 2020
Check for updates

(C) Author(s) (or their employer(s)) 2020. Re-use permitted under CC BY-NC. No commercial reuse. See rights and permissions. Published by BMJ.

To cite: Vasant DH, Hasan SS, Cruickshanks $\mathrm{P}$, et al. Frontline Gastroenterology Epub ahead of print: [please include Day Month Year]. doi:10.1136/ flgastro-2020-101679

\section{ABSTRACT}

Objective Severe irritable bowel syndrome (IBS) in school children and adolescents often leads to stigmatisation, social withdrawal, disrupted education and psychological distress. While there are few effective treatment options for IBS in this age group, gut-focused hypnotherapy (GFH) has shown promise in several trials. Unfortunately, GFH is not widely available, and clinical data outside of trials are scarce. Here, we evaluated outcomes from GFH in patients with IBS, aged $\leq 18$ years, from a tertiary referral centre.

Design/Method Consecutive patients aged $\leq 18$ years with severe IBS received 12 sessions of $\mathrm{GFH}$, at weekly intervals, using the Manchester Protocol. Clinical outcomes data, including IBS Symptom Severity Score (IBS-SSS), Hospital Anxiety and Depression Scale (HADS), Non-colonic Symptom Score and Quality-of-Life (QoL) score, were collected prospectively, and compared preGFH and post-GFH. Clinical response was defined as $\geq 50$ point reduction in IBS-SSS.

Results 32 young patients fulfilling Rome III diagnostic criteria for IBS (median age 16 (range 8-18) years, $n=23 / 32$ (72\%) female individuals) completed GFH. At baseline, the mean duration of IBS was $5.9 \pm 0.9$ years, and the mean IBS-SSS was $313 \pm 14$. After $\mathrm{GFH}, 28 / 32$ (88\%) responded, with a mean overall reduction in IBS-SSS $-159 \pm 16$ $(p<0.0001)$, and 24/32 (75\%) achieved $\geq 30 \%$ reduction in abdominal pain scores. GFH also improved: non-colonic symptoms ( $p<0.0001)$, HADS-anxiety $(p<0.0001)$, HADS-depression $(p=0.0002)$ and QoL Scores $(p<0.0001)$. Conclusion GFH is highly effective in children and adolescents with IBS. Early intervention with GFH in childhood IBS may reduce the subsequent burden of this problem in adults.

\section{INTRODUCTION}

Functional gastrointestinal disorders (FGIDs) such as irritable bowel syndrome (IBS) are common in children and adolescents, affecting approximately $25 \%$ of

\section{Significance of this study}

What is already known on this topic

- Irritable bowel syndrome (IBS) in children and adolescents disrupts education, causes embarrassment, is stigmatised and often progresses into adulthood.

- While there are few effective IBS treatments for children, hypnotherapy has shown promise in clinical trials. Unfortunately, uptake is hampered by scepticism and the lack of 'real-world' clinical data.

What this study adds

- This study suggests that hypnotherapy is highly effective for IBS in children and adolescents with $88 \%$ responding.

- This study demonstrates that, like adults, children and adolescents with IBS suffer debilitating non-colonic symptoms, which also respond to hypnotherapy.

How might it impact on clinical practice in the foreseeable future

- Due to there being even fewer treatment options in this age group, hypnotherapy could be considered at an earlier stage than in adults, with the potential to prevent adult suffering.

the population, and are associated with impaired quality of life (QoL). ${ }^{1-3}$ IBS is the most common cause of recurrent, 'non-organic' abdominal pain in this age group. ${ }^{4}$ Unfortunately, school children with chronic bowel disorders such as IBS often experience teasing, ${ }^{5}$ embarrassment $^{6}$ and stigma, which can result in social withdrawal. ${ }^{7}$ Moreover, recurrent chronic abdominal pain can significantly disrupt education, ${ }^{8}$ result in significant healthcare utilisation and socioeconomic costs, ${ }^{9}$ and many cases will progress and transition into adult IBS services. ${ }^{10}$ 
Disappointingly, the efficacy of pharmacological therapy for IBS in those aged $\leq 18$ years is low. ${ }^{11}$ While antidepressants are among the mainstay of treatment in adult IBS, ${ }^{12}$ their evidence base is fairly limited in children, ${ }^{11}$ and use in this population is often limited by safety concerns, with a reported increased risk of suicide. ${ }^{13}$ The lack of efficacious medical options makes behavioural and psychological therapies such as gut-focused hypnotherapy (GFH) attractive. Indeed GFH has been shown to be highly effective in IBS in adult populations, ${ }^{14}{ }^{15}$ with a response rate of $76 \%$ in the largest study to date ${ }^{16}$ with reduced healthcare utilisation. In children and adolescents with IBS, clinical trial data have shown that GFH is significantly superior to standard medical care in inducing clinical remission, ${ }^{17-19}$ with long-term effects, maintained at 5 years post-treatment. ${ }^{20}$ These promising data suggest that GFH could be considered as a first-line option for IBS in children ${ }^{21}$; however, clinical outcomes' data in patients aged $\leq 18$ years outside of clinical trials are lacking. ${ }^{22}$ Here, we evaluated outcomes from GFH in patients aged $\leq 18$ years of age referred to a tertiary referral centre for FGIDs.

\section{METHODS}

\section{Patient population}

The hypnotherapy unit in South Manchester has been treating patients with refractory FGIDs for over 35 years and is an established tertiary referral centre for these cases. GFH is endorsed by the National Institute for Clinical Excellence when conventional treatments have failed. ${ }^{23}$ Despite being set up as an adult service, due to the high response rates, there has been an increasing number of children and adolescents referred to our service with refractory IBS from paediatric services to consider GFH.

\section{Inclusion criteria}

Consecutive young patients (aged $\leq 18$ years) with IBS refractory to conventional management strategies, treated in the hypnotherapy unit at Wythenshawe Hospital, Manchester University National Health Service Foundation Trust, between November 2015 and 2019, were included in this study.

Prior to recommending GFH, all patients referred were reviewed and screened by a consultant gastroenterologist in the tertiary clinic to confirm the diagnosis of IBS according to Rome III diagnostic criteria, ensure conventional treatments have been exhausted, and to assess suitability for GFH.

\section{Exclusion criteria}

As in adults, the main exclusion criteria for GFH included those with major psychopathologies ${ }^{14}$ or children and adolescents with severe learning disabilities who would not be able to understand or engage with the treatment.
Data collection

Clinical outcomes data were collected prospectively. All included patients met the Rome III diagnostic criteria for IBS. At baseline, all patients completed the Tellegen absorption scale ${ }^{24}$ as a measure of hypnotic ability, where scores of 26-34 would be considered high, 16-25 medium and $\leq 15$ low.

\section{Outcome measures}

Questionnaire data for the following outcome measures were collected prospectively from all patients at baseline and after GFH.

\section{IBS Symptom Severity Score}

This score, which is now universally used in IBS clinical trials and is increasingly used in routine clinical practice, was the primary endpoint for this study. It consists of five domains (pain severity, pain frequency, abdominal bloating, bowel habit dissatisfaction and life interference), each of which scores a maximum of 100. Composite IBS Symptom Severity Scores (IBSSSSs) can be used to classify patients into mild $(<175)$, moderate (175-300) and severe (>300) IBS, and a 50 point reduction in IBS-SSS indicates a clinically significant improvement. ${ }^{25}$ Hence, the primary endpoint in this study was the percentage of patients achieving a clinical response defined by $\geq 50$ point reduction in total IBS-SSS. As a secondary endpoint, the Federal Drug Administration's recommended endpoint of $\geq 30 \%$ improvement in abdominal pain scores ${ }^{26}$ was also evaluated.

Non-colonic Symptom Score

As previously described, ${ }^{16}$ this consists of 10 items (nausea/vomiting, early satiety, headaches, backache, lethargy, excess wind, heartburn, urinary symptoms, thigh pain and aches, and pains in muscles and joints (bodily aches)), each scoring up to a maximum of 100 , the sum of which is divided by 2 to give a maximum score of $500 .^{27} 28$

\section{QoL Score}

This score consists of 15 components, coping with problems, confidence and security, quality of sleep, feelings of irritability, frequency of worrying, enjoyment of life, feelings of hopefulness, physical wellbeing, relationships with others, maintaining friendships, feelings of inferiority, feeling wanted, feelings of helplessness, difficulty in making decisions and enjoyment of leisure time. Each item is scored 0-100. As in previously published studies, the sum of these 15 components is divided by 3 to give a maximum QoL Score of $500 .{ }^{16}$ The higher the score, the better the QoL. ${ }^{1628}$

\section{Hospital Anxiety and Depression Scale}

The Hospital Anxiety and Depression Scale (HADS) has been used extensively as an outcome measure in 
previous GFH studies. ${ }^{162-30}$ It consists of anxiety and depression questions, with a maximum overall score for either domain of 21 . As in previous GFH studies, a cut-off of $\geq 10$ was used for clinical anxiety and depression. $^{16}$

\section{Procedure}

All patients received 12 sessions of GFH, each lasting up to $60 \mathrm{~min}$, at weekly intervals, over 3 months. All patients were treated by one of the two clinical hypnotherapists ( $\mathrm{SHH}$ and $\mathrm{PC}$ ), both of whom have a specialist interest and are vastly experienced in treating patients aged $\leq 18$ years. No new medical, dietary or other psychological interventions were commenced during the period of GFH. Patients were treated using the Manchester protocol which has been described in detail elsewhere, ${ }^{14}$ the aims of which are to induce a deep state of relaxation to guide the patient to learn how to control their gut function. The structure of the programme includes an introductory session. This first session is devoted to education and explanation about IBS, what the treatment involves, and how the use of imagery and tactile methods during GFH can help improve the patient's symptoms. In children and adolescents, this first session is particularly important in tailoring a developmentally appropriate approach for the subsequent treatment sessions, to maximise the ability to engage in the GFH treatment process. During this consultation session, the therapist first elicits how the condition affects the patient and the impact that the physical suffering has on their quality of life, for example, home, social and school (or work). The therapist explains how the gut functions and the impact of IBS on how the gut works, often using diagrams and drawings, as well as explanations. This approach often works very well with younger children who may also find that they can explain more easily through drawings what they think is happening in their gut. During this assessment, the therapist will discuss with the child any factors that make their condition better or worse. Other relevant information to guide the subsequent treatment sessions includes finding out about hobbies and interests the child/young person has, for example, favourite stories, toys, games, sport and nature so that these can be incorporated into the treatment to enhance their experience and improve engagement. The treatment is further adapted according to the child/young person's stage of development, for example, often the idea of imagining the gut as a river and the patient can 'slow it down' or 'speed it up' depending on their symptoms. While older children often find this analogy very helpful, younger children may find it more difficult to relate to this, and using stories as an alternative can therefore often be more useful for the younger child. The child/young person is usually accompanied by a parent for the consultation, which enables the parent to understand the treatment too. Some of the young patients prefer a parent to be present during treatment, which can be accommodated without any difficulty, and parents can often be helpful in helping the child/young person explain their problems. The treatment process is very much a partnership with the child/young person, and the patient is encouraged to develop ideas that they like so the work becomes very much their own.

During subsequent sessions, the child/young person sits or lies down in a comfortable reclining chair, and hypnotherapy is induced in a standard way by eye closure, progressive muscular relaxation and standard deepening techniques. The therapist uses a very soothing voice like one would use when reading a bedtime story. Then by incorporating the things they are interested in and that are appropriate for them to imagine, as discussed during the initial consultation visit, such concepts are used to focus on helping the gut to get better. Other important techniques during GFH include the child/young person placing a hand on their 'tummy', which is very popular during sessions and is often imagined as 'warming and soothing their tummy', which can enhance the process when they can feel the warmth, and then they can use this at will. The language used throughout would be adapted according to the age of the patient. As the course of treatment progresses, increasing emphasis is placed on controlling gut function, with the ultimate aim of enabling the patient to be 'in control of their gut' rather than the gut controlling them. ${ }^{14}$ The therapists often adapt the therapeutic approach according to the patients' symptoms and personal imagery of the condition to stimulate engagement, which often improves response, as well as improving the child/young person's confidence. The child/adolescent is provided with a recording of the sessions on a $\mathrm{CD}$ and are asked to practise the techniques daily, which they are advised to continue to use after completion of treatment.

\section{Ethical statement}

All the questionnaires used in this study are used routinely in the department to monitor the patient's progress. Consequently, it was considered that this study was a service evaluation of clinical outcomes from our existing service, and therefore the ethical review was not necessary.

\section{Statistical analysis}

Questionnaire data were digitalised on a Microsoft Excel spreadsheet and analysed to determine the percentages of patients that responded, and achieved $\geq 30 \%$ improvement in abdominal pain scores, following GFH. Data are expressed as means $( \pm \mathrm{SE}$ of the mean (SEM)) unless otherwise stated. Mean outcomes' data, including IBS-SSS, Non-colonic Symptom Scores, QoL Scores, HADS depression and anxiety scores, were compared before and after GFH using paired t-tests using a standard statistical software package (Stats Direct V.3.1.1, UK). P values $\leq 0.05$ 


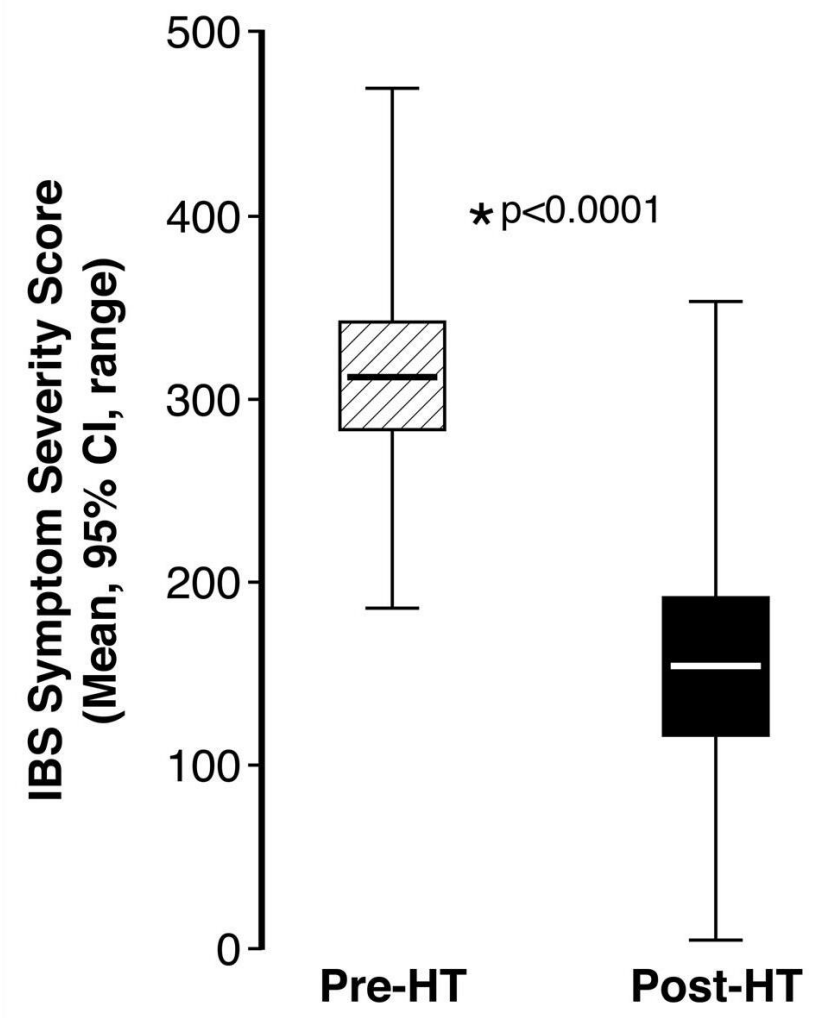

Figure 1 Improvement in IBS symptom severity post HT. HT, hypnotherapy; IBS, irritable bowel syndrome.

were considered statistically significant (only twosided $\mathrm{p}$ values have been displayed).

\section{RESULTS}

32 patients (mean age $15.7 \pm 0.4$ years, range $8-18$ ), with a mean duration of IBS $5.9 \pm 0.9$ years, were studied. Thirteen (41\%) patients had diarrhoeapredominant IBS (IBS-D), 9 (28\%) had constipationpredominant IBS and 10 (31\%) had an alternating bowel habit (IBS-alt). Based on baseline IBS-SSS data, 1 patient (3\%) had mild IBS, $14(44 \%)$ had moderate IBS and 17 (53\%) had severe IBS. The mean Tellegen absorption scale score was $16.8 \pm 1.5$. All 32 patients completed the full course of GFH, and all patients were successfully hypnotised during each of their 12 sessions.

\section{Overall results}

IBS Symptom Severity Scores

The mean baseline IBS-SSS was $313 \pm 14$. After hypnotherapy, $28(88 \%)$ patients achieved a clinically significant response ( $\geq 50$ point reduction in IBS-SSS). Moreover, the majority achieved the more demanding endpoints of $\geq 100$ point reduction $(n=26,81 \%)$ and $\geq 150$ point reduction $(n=19,59 \%)$ in IBS-SSS. Overall, compared with baseline, there was a significant mean reduction in IBS-SSS post-GFH (change: -159.2, $\mathrm{p}<0.0001$, (95\% CI: 127-191; figure 1).

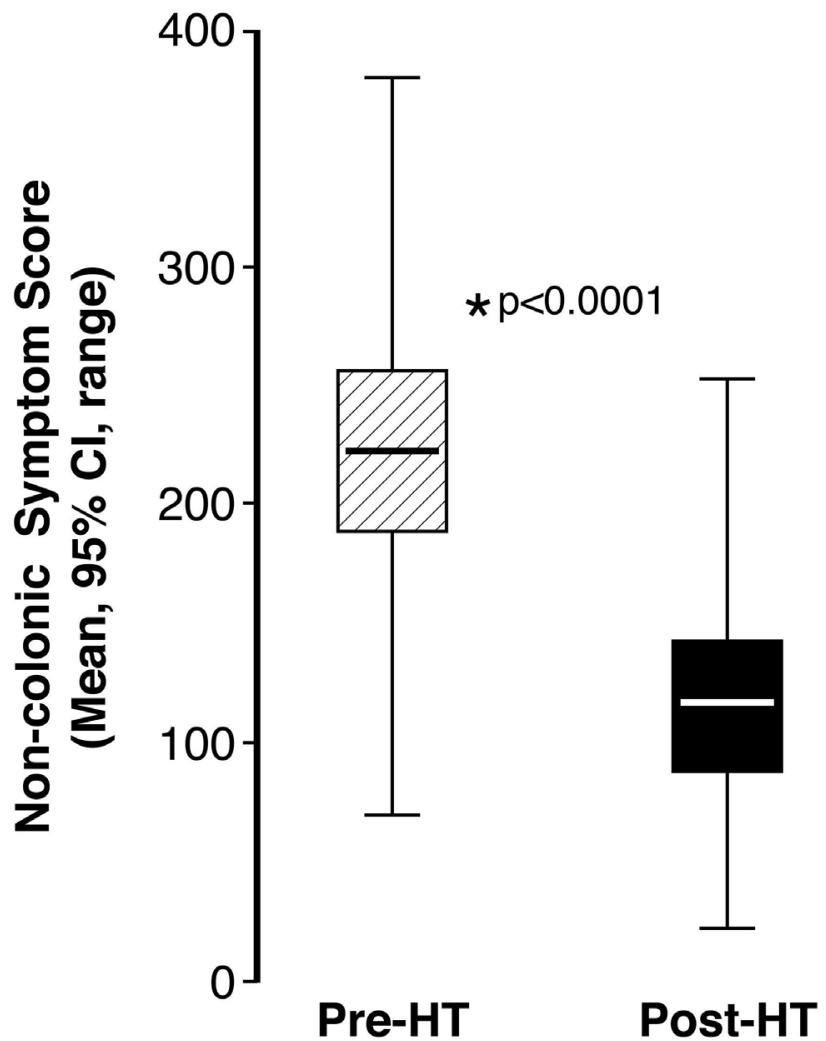

Figure 2 Improvement in Non-colonic Symptom Scores post HT. HT, hypnotherapy.

Hypnotic ability according to Tellegen absorption scale scores did not vary between responders achieving $\geq 50$ point reduction in IBS-SSS, compared with those who did not respond to GFH (Mann-Whitney U test: median 17.5 vs $17, \mathrm{U}=52, \mathrm{p}=0.81$ ).

There were highly significant mean reductions in the scores in all domains of IBS-SSS: pain severity scores (pre-GFH 61 vs 26 post-GFH, p<0.001), pain frequency scores (pre-GFH 61 vs 31 post-GFH, $\mathrm{p}<0.001$ ), abdominal bloating severity (pre-GFH 51 vs 26 post-GFH, $\mathrm{p}<0.0001$ ), dissatisfaction with bowel habit (pre-GFH 63 vs 33 post-GFH, $\mathrm{p}<0.0001$ ) and interference with life (pre-GFH 76 vs 39 post-GFH, $\mathrm{p}<0.0001)$.

\section{Non-colonic symptoms}

There was a highly significant reduction in mean noncolonic symptom scores after GFH (pre-GFH 220.3 vs post-GFH 115.1, $\mathrm{p}<0.0001,(95 \% \mathrm{CI})$ change 105.2 (78.7-131.8); figure 2). All components of the score were significantly reduced (table 1 ).

\section{Quality of life}

There was significant improvement in total QoL Score from a mean $267.7 \pm 14.3$ pre-GFH to $360.8 \pm 13.8$ post-GFH $(\mathrm{p}<0.0001$; mean $(95 \% \mathrm{CI})$ change 93.1 (68.8-117.4)), (online supplemental figure 1). Most individual components of the QoL Score significantly improved following GFH (table 2). 
Table 1 Mean non-colonic symptom scores before and after $\mathrm{GFH}$

\begin{tabular}{llll}
\hline & $\begin{array}{l}\text { Mean } \\
\text { symptom } \\
\text { score pre- }\end{array}$ & $\begin{array}{l}\text { Mean } \\
\text { symptom } \\
\text { score post- }\end{array}$ & \\
Non-colonic symptom & GFH & GFH & P value \\
\hline Nausea/ vomiting & $37.6 \pm 5.5$ & $12.8 \pm 3.2$ & $<0.0001$ \\
Early satiety & $33.3 \pm 6.1$ & $19.9 \pm 4.4$ & 0.01 \\
\hline Headaches & $46.6 \pm 5.6$ & $27.7 \pm 4.5$ & 0.0006 \\
\hline Backache & $45.3 \pm 6.5$ & $23.3 \pm 4.5$ & $<0.0001$ \\
Lethargy & $71.7 \pm 5.0$ & $42.7 \pm 4.8$ & $<0.0001$ \\
Excess wind & $68.7 \pm 5.5$ & $35.4 \pm 5.0$ & $<0.0001$ \\
Heartburn & $29.2 \pm 4.8$ & $11.5 \pm 3.3$ & $<0.0001$ \\
Urinary symptoms & $35.4 \pm 6.0$ & $20.5 \pm 5.1$ & 0.004 \\
Thigh pain & $21.0 \pm 4.7$ & $11.4 \pm 4.0$ & 0.03 \\
Musculoskeletal aches & $50.9 \pm 6.5$ & $25.2 \pm 4.4$ & $<0.0001$ \\
and pains & & & \\
\hline
\end{tabular}

GFH, gut-focused hypnotherapy.

\section{Anxiety and depression scores}

GFH significantly improved mean anxiety $(11.3 \pm 0.8$ vs $8.1 \pm 0.8, \mathrm{p}<0.0001)$ and depression scores (5.1 \pm 0.7 vs $2.8 \pm 0.4 ; p=0.0002$; online supplemental figure 2). Twenty-one patients (66\%) met the cut offs for clinical anxiety or depression (21/32 had HADS-A $\geq 10$ and $1 / 32$ had HADS-D $\geq 10$ ). Response rates to GFH and mean improvement in IBS-SSS post GFH did not differ between those with anxiety/depression and those without (response rates to GFH: anxiety/ depression 18/21, 86\% vs 10/11, 91\%, those without anxiety/depression, Fisher exact test $\mathrm{p}>0.99$; mean improvement in IBS-SSS: 165.8 anxiety/depression vs 150.8 those without anxiety/depression, unpaired t-test: $95 \% \mathrm{CI},-52.7$ to $82.7 ; \mathrm{p}=0.65)$.

\begin{tabular}{|c|c|c|c|}
\hline $\begin{array}{l}\text { Component of QoL } \\
\text { score }\end{array}$ & $\begin{array}{l}\text { Mean pre- } \\
\text { GFH }\end{array}$ & $\begin{array}{l}\text { Mean post- } \\
\text { GFH }\end{array}$ & $P$ value \\
\hline Coping with problems & $45.7 \pm 4.3$ & $74.7 \pm 3.9$ & $<0.0001$ \\
\hline Confidence and security & $43.8 \pm 4.6$ & $71.5 \pm 3.9$ & $<0.0001$ \\
\hline Quality of sleep & $61.2 \pm 4.9$ & $72.3 \pm 3.6$ & 0.008 \\
\hline Irritability & $37.7 \pm 5.5$ & $66.8 \pm 3.8$ & $<0.0001$ \\
\hline Frequency of worrying & $36.3 \pm 5.4$ & $56.9 \pm 5.3$ & 0.003 \\
\hline Enjoyment of life & $60.1 \pm 4.2$ & $75.1 \pm 3.5$ & 0.0005 \\
\hline Feelings of hopefulness & $58.6 \pm 4.7$ & $74.1 \pm 3.9$ & 0.0001 \\
\hline Physical well-being & $49.6 \pm 3.9$ & $74.6 \pm 3.1$ & $<0.0001$ \\
\hline Relationships with others & $70.6 \pm 4.2$ & $81.6 \pm 2.7$ & 0.008 \\
\hline Maintaining friendships & $72.0 \pm 4.8$ & $77.7 \pm 4.0$ & 0.13 \\
\hline Inferiority & $57.3 \pm 4.7$ & $73.8 \pm 4.6$ & 0.001 \\
\hline Feeling wanted & $58.4 \pm 5.4$ & $71.7 \pm 4.1$ & 0.01 \\
\hline Helplessness & $46.7 \pm 4.3$ & $70.1 \pm 4.3$ & $<0.0001$ \\
\hline $\begin{array}{l}\text { Difficulty in making } \\
\text { decisions }\end{array}$ & $48.1 \pm 4.5$ & $62.0 \pm 4.5$ & 0.0005 \\
\hline Enjoyment of leisure time & $66.1 \pm 3.9$ & $78.5 \pm 3.4$ & 0.004 \\
\hline
\end{tabular}

GFH, gut-focused hypnotherapy; QoL, quality of life.

\section{DISCUSSION}

This study has provided much needed 'real-world' clinical outcomes data on GFH in children and adolescents with IBS, and suggests that this treatment can be highly effective in this age group. The findings also demonstrate a similar burden of extraintestinal features of IBS to those seen in adult patients. These bothersome non-colonic symptoms including nausea, early satiety, headaches, backache, lethargy, excess wind, heartburn, urinary symptoms, thigh pain and musculoskeletal pains, undoubtedly contribute to considerable suffering, embarrassment, and may result in difficulty performing in school activities including physical education. Interestingly, the data presented here have shown significant improvement in all colonic and non-colonic symptoms following GFH. Many of our patients following GFH were able to return to fulltime education following a period of disrupted attendance. While measures of school attendance and parent perception were not obtained prospectively in our study, improvements in school attendance have previously been reported elsewhere in clinical trials of GFH (17-18). While the exact reasons why GFH improves school attendance remain unclear, based on our data, improvement in these debilitating and wide-ranging extraintestinal symptoms may be an important factor.

This study has demonstrated a highly significant improvement in the entire spectrum of IBS symptoms following hypnotherapy in children and adolescents. Interestingly, the overall response rate of $88 \%$ using IBS-SSS is even higher than the previously reported response rates to $\mathrm{GFH}$ in adult patients $(76 \%)$, using the same hypnotherapy protocols and same outcome measures. ${ }^{16}$ While the exact reasons for higher response rates in children and adolescents, when compared with adults, are unclear, this may plausibly relate to their increased receptivity to use of their imagination using multisensory imagery compared with older populations. ${ }^{31}$ All 32 patients in this study were successfully hypnotised during each session without any difficulty. This increased receptivity to this form of treatment and enhanced imagination allows the therapist to use the imagery even more effectively to work on strategies for problem resolution, developing self-regulation skills in the process. Compared with adults, children and adolescents undergoing GFH often need very little encouragement to practise the techniques learnt during the sessions at home using the recordings provided and are very quick to learn the technique of self-hypnosis. Furthermore, because a child has not had the problem for as long as an adult, they are less likely to be so entrenched in their illness, possibly making it more amenable to hypnotherapy. ${ }^{21}$

Our data strongly support GFH as a treatment for IBS in children and adolescents. Due to there being even fewer treatment options in this age group than adults, and based on our findings, arguably GFH should be considered at an earlier stage than in adults. 
Unfortunately, while prospective long-term follow-up data are not available for our cohort, previous clinical trials data in children and adolescents have shown longlasting effects, ${ }^{2032} 33$ and therefore, GFH may have the potential to prevent adult suffering. Moreover, the QoL Scores show a highly significant improvement overall and in almost all of the individual subscores. While clinical levels of anxiety affected around twothirds of our cohort, with significant improvements in both anxiety and depression levels after GFH, the beneficial effects seen on IBS symptoms were independent of whether or not the patients were anxious or depressed at baseline. Furthermore, the fact that all 32 patients completed all 12 weeks of therapy suggests that this treatment is both feasible and acceptable in young patients with refractory IBS.

Unfortunately, GFH is not currently widely available and continues to be surrounded by much scepticism. This is reflected by a recent survey of 289 Australian Paediatricians which disappointingly reported that only $2.1 \%$ would consider referral for hypnotherapy for a child or adolescent with IBS. ${ }^{34}$ Interestingly, the attitudes towards hypnotherapy referrals did not differ between those who believed there 'is clear evidence for effective treatments for IBS' and those 'who believed there is no clear evidence for effective IBS treatments'. ${ }^{34}$ Potential reasons for not considering GFH as a treatment for IBS, prior to our current study, may be due to the lack of previously published real-world clinical data. Other potential reasons may be a lack of access and availability of appropriately trained therapists, lack of knowledge and the need for further education. While recent adult literature publications have focused on the group $^{35}$ and telemedicine delivery of GFH for IBS $^{36}$ to improve access to this effective form of treatment, paediatric and adolescent data have tended to focus on non-therapist delivered self-therapy using recordings, with some success. ${ }^{37} 38$ One of the disadvantages of non-therapist delivered GFH is that the treatment delivered is generic, which may not make it suitable for refractory patients with severe functional impairment seen in tertiary care such as the patients included in our study. Patients with IBS and severe functional impairment often require an individualised approach. While face-to-face GFH is more costly, it is our experience that those with higher symptom severity scores tend to respond better to individualised GFH presumably because they suffer from a more complex form of the condition with additional psychological factors contributing. ${ }^{14}$ Recently, as an alternative to face-to-face GFH, we have been providing the option of remote individualised GFH via video consultation, with similar clinical efficacy, ${ }^{36}$ which has significantly improved access to therapy for patients, especially those living further afield from the treatment centre. This may also prove to be an excellent option for children and adolescents across different age groups, providing they have the appropriate parental support where needed, and access to the video technology.

One of the main limitations of our study is that it is a 'real-world' study of clinical outcomes, as opposed to a randomised controlled trial. While there was no control group in this study because all referrals for GFH have to be made in accordance with stringent NICE guidelines which reserve GFH for only refractory cases, ${ }^{23}$ all patients included in our study had already exhausted all conventional medical and dietary options to qualify for their treatment. This was mandatory because individualised funding requests for GFH were only approved by Clinical Commissioning Group (CCG) panels if these criteria were satisfied. All patients in our study had therefore tried and failed all viable treatment options including antidiarrhoeals and laxatives (where appropriate), antispasmodics, and the low Fermentable Oligo-, Di-, Mono-saccharides And Polyols (low-FODMAP) diet. The notable exception to the application of the adult NICE IBS guidelines in our series was the use of antidepressants, which were not used in any patient in our study. This was justified and agreed by the CCG panels, given that antidepressants are advised against by NICE in children and young people without prior use of psychological therapies, ${ }^{39}$ amidst safety concerns including the reported increased risk of suicide in this age group. ${ }^{13}$ Consequently, there was limited scope for deployment of any new therapies during the 12-week course of GFH. To avoid any confounding factors which could affect response to GFH, medication changes were not recommended by the gastroenterologists in any patient in this series during the course of treatment until follow-up after completion of GFH. This makes the chances of any confounding treatments extremely low.

\section{CONCLUSION}

In conclusion, GFH is a highly effective treatment for severe IBS in children and adolescents. GFH significantly improves both colonic and non-colonic symptoms, QoL and psychological distress levels. Paediatric and adult clinicians looking after young patients with refractory IBS should seriously consider referral for GFH.

Contributors DHV conceptualised the study, analysed and interpreted data, wrote the paper, and is the guarantor of the manuscript, SSH and PC acquired data and helped write the manuscript, PJW helped write the manuscript and provided important intellectual input.

Funding The authors have not declared a specific grant for this research from any funding agency in the public, commercial or not-for-profit sectors.

Competing interests None declared.

Patient consent for publication Not required.

Provenance and peer review Not commissioned; externally peer reviewed.

Data availability statement All data relevant to the study are included in the article or uploaded as supplementary information. 
Supplemental material This content has been supplied by the author(s). It has not been vetted by BMJ Publishing Group Limited (BMJ) and may not have been peer-reviewed. Any opinions or recommendations discussed are solely those of the author(s) and are not endorsed by BMJ. BMJ disclaims all liability and responsibility arising from any reliance placed on the content. Where the content includes any translated material, BMJ does not warrant the accuracy and reliability of the translations (including but not limited to local regulations, clinical guidelines, terminology, drug names and drug dosages), and is not responsible for any error and/or omissions arising from translation and adaptation or otherwise.

Open access This is an open access article distributed in accordance with the Creative Commons Attribution Non Commercial (CC BY-NC 4.0) license, which permits others to distribute, remix, adapt, build upon this work noncommercially, and license their derivative works on different terms, provided the original work is properly cited, appropriate credit is given, any changes made indicated, and the use is noncommercial. See: http://creativecommons.org/licenses/by-nc/4. $0 /$.

\section{ORCID iD}

Dipesh H Vasant http://orcid.org/0000-0002-2329-0616

\section{REFERENCES}

1 Lewis ML, Palsson OS, Whitehead WE, et al. Prevalence of Functional Gastrointestinal Disorders in Children and Adolescents. J Pediatr 2016;177:39-43. e3.

2 Saps M, Velasco-Benitez CA, Langshaw AH, et al. Prevalence of functional gastrointestinal disorders in children and adolescents: comparison between Rome III and Rome IV criteria. J Pediatr 2018;199:212-6.

3 Robin SG, Keller C, Zwiener R, et al. Prevalence of pediatric functional gastrointestinal disorders utilizing the Rome IV criteria. J Pediatr 2018;195:134-9.

4 Boronat AC, Ferreira-Maia AP, Matijasevich A, et al. Epidemiology of functional gastrointestinal disorders in children and adolescents: a systematic review. World J Gastroenterol 2017;23:3915.

5 Quick V, McWilliams R, Byrd-Bredbenner C. A case-control study of current psychological well-being and weight-teasing history in young adults with and without bowel conditions. $J$ Hum Nutr Diet 2015;28:28-36.

6 Thomson S, Dancey CP. Symptoms of irritable bowel in school children: prevalence and psychosocial effects. J Pediatr Health Care 1996;10:280-5.

7 Hearn M, Whorwell PJ, Vasant DH. Stigma and irritable bowel syndrome: a taboo subject? Lancet Gastroenterol Hepatol 2020;5:607-15.

8 Størdal K, Nygaard EA, Bentsen BS. Recurrent abdominal pain: a five-year follow-up study. Acta Paediatr 2005;94:234-6.

9 Hoekman DR, Rutten JMTM, Vlieger AM, et al. Annual costs of care for pediatric irritable bowel syndrome, functional abdominal pain, and functional abdominal pain syndrome. $J$ Pediatr 2015;167:1103-8. e2.

10 Gieteling MJ, Bierma-Zeinstra SMA, Passchier J, et al. Prognosis of chronic or recurrent abdominal pain in children. $J$ Pediatr Gastroenterol Nutr 2008;47:316-26.

11 Devanarayana NM, Rajindrajith S. Irritable bowel syndrome in children: current knowledge, challenges and opportunities. World J Gastroenterol 2018;24:2211-35.

12 Ford AC, Lacy BE, Harris LA, et al. Effect of antidepressants and psychological therapies in irritable bowel syndrome: an updated systematic review and meta-analysis. Am J Gastroenterol 2019;114:21-39.

13 Spielmans GI, Spence-Sing T, Parry P. Duty to warn: antidepressant black box suicidality warning is empirically justified. Front Psychiatry 2020;11:18.

14 Vasant DH, Whorwell PJ. Gut-focused hypnotherapy for functional gastrointestinal disorders: evidence-base, practical aspects, and the Manchester protocol. Neurogastroenterol Motil 2019;31:e13573.

15 Black CJ, Thakur ER, Houghton LA, et al. Efficacy of psychological therapies for irritable bowel syndrome: systematic review and network meta-analysis. Gut 2020;69:1441-51.

16 Miller V, Carruthers HR, Morris J, et al. Hypnotherapy for irritable bowel syndrome: an audit of one thousand adult patients. Aliment Pharmacol Ther 2015;41:844-55.

17 Vlieger AM, Menko-Frankenhuis C, Wolfkamp SCS, et al. Hypnotherapy for children with functional abdominal pain or irritable bowel syndrome: a randomized controlled trial. Gastroenterology 2007;133:1430-6.

18 Weydert JA, Shapiro DE, Acra SA, et al. Evaluation of guided imagery as treatment for recurrent abdominal pain in children: a randomized controlled trial. BMC Pediatr 2006;6:29.

19 van Tilburg MAL, Chitkara DK, Palsson OS, et al. Audiorecorded guided imagery treatment reduces functional abdominal pain in children: a pilot study. Pediatrics 2009;124:e890-7.

20 Vlieger AM, Rutten JMTM, Govers AMAP, et al. Long-Term follow-up of gut-directed hypnotherapy vs. standard care in children with functional abdominal pain or irritable bowel syndrome. Am J Gastroenterol 2012;107:627-31.

21 Whorwell PJ. Hypnotherapy: first line treatment for children with irritable bowel syndrome? Arch Dis Child 2013;98:243-4.

22 Agrawal A, Whorwell PJ. Hypnotherapy for irritable bowel syndrome: a role in pediatric practice? J Pediatr Gastroenterol Nutr 2005;41 Suppl 1:S49-50.

23 NICE. Irritable bowel syndrome in adults: diagnosis and management National Institute for healthcare and excellence guidelines; CG61, 2008.

24 Tellegen A, Atkinson G. Openness to absorbing and selfaltering experiences ("absorption"), a trait related to hypnotic susceptibility. J Abnorm Psychol 1974;83:268-77.

25 Francis CY, Morris J, Whorwell PJ. The irritable bowel severity scoring system: a simple method of monitoring irritable bowel syndrome and its progress. Aliment Pharmacol Ther 1997;11:395-402.

26 FDA. Guidance for Industry: irritable bowel syndrome - clinical evaluation of drugs for treatment, 2012.

27 Whorwell PJ, McCallum M, Creed FH, et al. Non-colonic features of irritable bowel syndrome. Gut 1986;27:37-40.

28 Gonsalkorale WM, Houghton LA, Whorwell PJ. Hypnotherapy in irritable bowel syndrome: a large-scale audit of a clinical service with examination of factors influencing responsiveness. Am J Gastroenterol 2002;97:954-61.

29 Zigmond AS, Snaith RP. The hospital anxiety and depression scale. Acta Psychiatr Scand 1983;67:361-70.

30 Jones H, Cooper P, Miller V, et al. Treatment of noncardiac chest pain: a controlled trial of hypnotherapy. Gut 2006;55:1403-8.

31 Kohen DP, Kaiser P. Clinical hypnosis with children and Adolescents-What? why? how?: origins, applications, and efficacy. Children 2014;1:74-98.

32 Rexwinkel R, Vlieger A, Bovendeert J, et al. Long-Term followup of gut-directed hypnotherapy self-exercises at home using $\mathrm{CD}$ versus individual therapy by qualified therapists in children with irritable bowel syndrome or functional abdominal pain syndrome. J Pediatr Gastroenterol Nutr 2019;68:1.

33 Gonsalkorale WM, Miller V, Afzal A, et al. Long term benefits of hypnotherapy for irritable bowel syndrome. Gut 2003;52:1623-9.

34 Heinsch ML, Nightingale S. Functional gastrointestinal disorders in children and adolescents: knowledge, practice and attitudes of Australian paediatricians. J Paediatr Child Health 2019;55:1063-9. 
35 Flik CE, Laan W, Zuithoff NPA, et al. Efficacy of individual and group hypnotherapy in irritable bowel syndrome (IMAGINE): a multicentre randomised controlled trial. Lancet Gastroenterol Hepatol 2019;4:20-31.

36 Hasan SS, Pearson JS, Morris J, et al. SKYPE hypnotherapy for irritable bowel syndrome: effectiveness and comparison with face-to-face treatment. Int J Clin Exp Hypn 2019;67:69-80.

37 Rutten JMTM, Vlieger AM, Frankenhuis C, et al. Home-

Based hypnotherapy self-exercises vs individual hypnotherapy with a therapist for treatment of pediatric irritable bowel syndrome, functional abdominal pain, or functional abdominal pain syndrome: a randomized clinical trial. JAMA Pediatr 2017;171:470-7.

38 Gulewitsch MD, Schlarb AA. Comparison of gut-directed hypnotherapy and unspecific hypnotherapy as self-help format in children and adolescents with functional abdominal pain or irritable bowel syndrome: a randomized pilot study. Eur J Gastroenterol Hepatol 2017;29:1351-60.

39 NICE. Depression in children and young people: identification and management. NICE guideline [NG134], 2019. 